\title{
Implementation of Hybrid Indoor Positioning System based on Wi-Fi and PDR in Smartphone
}

\author{
Boney A. Labinghisa, Dong Myung Lee
}

\begin{abstract}
This paper proposed thehybridindoor positioning system in smartphone for positioning accuracy by fusion of wireless-fidelity (Wi-Fi) signals and inertial sensors from pedestrian dead reckoning (PDR) in smartphone. The proposed system uses Wi-Fi as the source of received signal strength indicator (RSSI) for fingerprint and smartphones sensor data from PDR. RSSI signals are used to determine the initial position and reduce error accumulation of PDR while smartphone sensor data are used to estimate user trajectory. Extended Kalman Filter (EKF) is the fusion algorithm used for its similarity with Kalman Filter (KF) but with advantages of processing non-linear progressions. An estimated 49 steps were detected which is identical to the 50 steps taken in the experiment while showing a trajectory similar to the actual route taken by the mobile user. A benefit of using built-in smartphone sensors is its cost-effectiveness and availability that does not require additional hardware. In addition, a nonlinear EKF is used to enhance the positioning accuracy in the proposed system. Further studies will be made in the potential of indoor positioning algorithm including the effect of noise interference on sensors and RSSI and the accumulated errors resulting from walking.
\end{abstract}

Keywords: Extended Kalman Filter, Fingerprinting, Indoor Positioning, PDR, Smartphone Sensors, Wi-Fi, RSSI

\section{INTRODUCTION}

In recent years, technological advances have been made in smartphone uses. Almost all smartphones available in the market have embedded sensors such as GPS, accelerometers, gyroscopes, magnetometers, proximity sensors and barometers [1]. Having these inertial sensors makes it possible to determine movement and orientation in three axes. Taking advantage of this feature can estimate the exact changes in the movement and position of a smartphone user. Pedestrian dead reckoning is one of the major positioning technology. However, using pedestrian dead reckoning (PDR) alone incurs errors in calibration that accumulates with time making it inefficient if used solely. Another indoor positioning technique is wireless-fidelity (Wi-Fi) fingerprinting but radio signals are also unreliable due to signal attenuation caused by non-line of sight (NLOS) obstacles in indoor spaces.

Both Wi-Fi and PDR positioning systems have their

Revised Manuscript Received on July 22, 2019.

Boney A. Labinghisa, Ph.D. student, Department of Computer Engineering, Tongmyong University, 48520 Busan, Republic of Korea. Email: blabinghisa@yahoo.com

Dong Myung Lee*, Professor, Department of Computer Engineering, Tongmyong University, 48520 Busan, Republic of Korea

Email: dmlee@tu.ac.kr algorithm that takes advantage of these inertial sensing

advantages and disadvantages with achieving accurate and precise positioning. Wi-Fi has an advantage by providing the absolute position of a user in an unobstructed indoor environment but often suffers from RSS fluctuations while PDR can give an estimation of moving users in a short period of time but also suffers from accumulated drift errors. Fusion of positioning systems has become popular such as that of Particle Filter (PF) and Kalman Filter (KF) but this have problems in implementation. PF requires higher computational resources which are not applicable on most smartphones and KF is only efficient on linear measurements. In order to fuse $\mathrm{Wi}-\mathrm{Fi}$ and PDR for optimizing positioning performance, extended Kalman Filter (EKF) is the most suited [2].

In this paper, thehybridindoor positioning system in smartphone is proposed to solve the individual problems of $\mathrm{Wi}-\mathrm{Fi}$ and PDR. The proposed system will combine the inertial measurement from PDR with the fingerprint map generated using receive signal strength indicator (RSSI) to correct the accumulated directional errors [3]. Finally, the aim of this paper is to reduce trajectory errors in PDR and avoid intermittent RSSI spikes that are the main concerns in reducing positioning accuracy.

\section{RELATED RESEARCHES}

Due to the advantages of smartphones with built-in sensors, it offers the ability to physically measure user step length and heading. The approach of considering PDR as a linear function creates inaccurate estimation and error accumulation [4]. The positioning technology using Wi-Fi does not also require additional hardware with measuring RSSI values from several wireless sources for collecting and creating a fingerprint map during offline phase, and matching with real-time RSSI samples during online phase. This complementary advantage makes it desirable to study more on the hybrid fusion of Wi-Fi and PDR.

The indoor positioning techniques using Wi-Fi has seen many approaches with low-cost, high accuracy, low-complexity, and robustness. In [5], the information on the physical layer in the scheme can be easily obtained in the Wi-Fi fingerprint scheme. The measured and obtained RSS reflects the distance information of the transmitter and the receiver. Because each position in an indoor environment receives a unique signal strength due to the multi-path effect, the signal property, especially the signal strength, has its own fingerprint. A fingerprint map is actually built up using this property. 
Wi-Fi fingerprint scheme has been a popular positioning technique since the idea of RADAR [6] and has since been improved with different approaches and ideas added to its concept. A study on the properties of Wi-Fi signals in finding the user position [7], was done, and it provided results that the certain factors may affect the properties of Wi-Fi signals that can affect the positioning. The accuracy of the positioning varies on the orientation of user or mobile unit, the temporal and spatial variations of Wi-Fi signals, the time dependency, the device hardware and a number of samples.

Instead of using particle filter, EKF was selected as the fusion algorithm because of its lesser burden in computation. The research used smartphone gyroscope sensor for heading estimation in the PDR system because of its advantages in the indoor environment of modern structures mostly composed of steel and concrete [8]. For obtaining the starting position in PDR, it is presented in several ways like Global Positioning System (GPS) tracking [9], identifying landmarks [10] and using RSSI in Wi-Fi fingerprint localization [11]-[12].

\section{PROPOSED HYBRIDINDOOR POSITIONING SYSTEM}

The proposed system is divided into the PDR and Wi-Fi modules which have each separated function but their results are integrated using EKF as the fusion algorithm. In order to achieve high accuracy and coping with the complexity of indoor environments, robust filter such as EKF is applied in the integrated Wi-Fi and PDR, and accumulated errors on observed and updated states can be reduced.

PDR uses the accelerometer for step detection and steps length while gyroscope and magnetometer determine the orientation and heading of the mobile device. Wi-Fi uses radio signals to make a map as a fingerprint of unique RSSI and stores it in a database, which is later used to match with real-time receive $\mathrm{Wi}-\mathrm{Fi}$ signals. The overall architecture of the proposed system is shown in Fig. 1 [13].

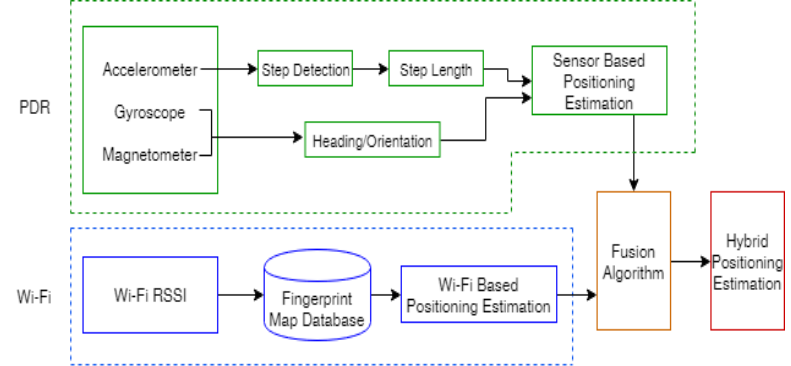

Fig. 1. Architecture of the proposed hybridindoor positioning system.

In theory, EKF is a non-linear version of $\mathrm{KF}$ which linearizes the current estimated trajectory mean and covariance. EKF is a mathematical-model-based optimal state estimator. The whole estimation of the system is conveyed by a mean state $X_{\mathbb{t}}$ approximate covariance matrix $P_{\mathbb{t}}$. These are used to predict the true value of the model. The estimated true value $\hat{x}_{t}$ is the prediction within the model that is likely the true state.

In comparison, unscented KF [14] uses deterministic sampling that computes the covariance matrices in EKF. EKF SLAM inconsistency cannot be solved in [16] but can be improved in [15]. Another comparable filter to EKF is particle filter which generates a random set of points known as particles that creates almost equivalent to direct sampling of the probability density function of interest [17]. The greater the number of particles, the results can achieve high accuracy in localization. Although particle filter shows a higher performance to EKF, the main drawback is the large size of the samples needed.

\section{A. Pedestrian Dead Reckoning}

EKF is also used to fuse $\mathrm{Wi}-\mathrm{Fi}$ and PDR because of its capability to determine current position using RSSI which sets the initial position necessary for PDR and continuously update the trajectory based on inertial measurements. In this paper, the PDR of mobile user has a state vector $X_{t}$, that includes 4 states at timet, $X_{t}=\left\lfloor x_{t a} y_{t^{3}} \varphi_{t a} v_{t}\right\rfloor$ where $\mathrm{x}$ and $\mathrm{y}$ are $2 \mathrm{D}$-coordinates of the position, $\emptyset$ is the orientation and $v_{t}$ is the velocity. Velocity is assumed to be $0 \mathrm{~m} / \mathrm{s}$ when the pedestrian is not moving and $0.68 \mathrm{~m} / \mathrm{s}$ when walking one step [18].

And, $P_{\mathbb{t}}$ is covariance matrix of the state, $\mathrm{Q}$ is the covariance matrix of the process state and $\mathrm{R}$ is covariance matrix of the observation noise at time $t$. Smartphones have built-in accelerometer and gyroscope sensors to provide input vector, $u_{t}=\left[v_{t s} w_{t}\right]$ at each time step, where $w_{t}$ is the yaw rate.

Step length $L_{s}$ : Can be computed using, $k$ as the constant value for unit conversion, $a_{i}$ and $N$ represent the vertical acceleration sample and the total number of samples in one step as seen in (1). Position of the current step can be determined by adding displacement from the previous step. $L=\left(x_{i d} y_{i}\right)$ and $L_{i-1}=\left(x_{i-1}, y_{i-1}\right)$ represents the current step and the step taken previously and $\emptyset$ is the heading angle derived from gyroscope and magnetometer as seen in(2).

$$
\begin{aligned}
& L_{g}=k \times \sqrt[3]{\sum_{i=1}^{N}\left|a_{i}\right| / N} \\
& L=L_{i-1}+L_{g} \times\left[\begin{array}{c}
\cos \emptyset \\
\sin \emptyset
\end{array}\right]
\end{aligned}
$$

\section{B. Wi-Fi Fingerprinting}

Wi-Fi fingerprint in Fig. 1 comprises of two phases: offline data training and online phases. An offline generates a fingerprint map database with RSSI values in reference points (RPs) acquired from access points (APs). In online phase, mobile user position is obtained by matching the real-time RSSI value with the fingerprint map. A fingerprint map can be represented as a table consisting of $\mathrm{x} \& \mathrm{y}$ coordinates in $2 \mathrm{D}$ plane, RPs, and $A P_{N}$ as seen in Table I. RPs can have a maximum number $i$ depending on the size and interval specified in the experiments. Each RP is assigned a coordinate, where $A P_{N} \mathrm{RSSI}$ is acquired and each will have a unique set of FP_RSSI.

Distance estimation $\mathrm{d}$ is summarized using Euclidean distance in (3), where $R S S I_{i}$ is $i^{\text {th }}$ real-time measured $R S S I$ signal during online phase and $F P_{-} R S S I_{i}$ is $i^{\text {th }}$ RSSI value measured from the fingerprint map during offline phase. This will determine the position in 2D-coordinates as $x \& y$ information of mobile user's 
position, $\mathrm{z}_{\mathrm{t}}=\left[\mathrm{x}_{\mathrm{t},} \mathrm{y}_{\mathrm{t}}\right]$ observed at each interval.

$$
d=\sqrt{\sum_{i=1}^{N}\left(R S S I_{i}-F P_{-} R S S I_{i}\right)^{2}}
$$

Table I: Table representing a fingerprint map of RSSI and APs

\begin{tabular}{|c|c|c|c|c|c|}
\hline$R P$ & $\mathbf{x}, \mathbf{y}$ & $A P_{1}$ & $A P_{2}$ & $x$ & $A P_{N}$ \\
\hline 1 & $\mathrm{x}_{1}, \mathrm{y}_{1}$ & $F P \_R S S I_{11}$ & $F P_{-} R S S I_{12}$ & $\mathrm{max}$ & $F P_{-} R S S I_{l, N}$ \\
\hline 2 & $\mathrm{x}_{2}, \mathrm{y}_{2}$ & $F P \_R S S I_{21}$ & $F P \_R S S I_{22}$ & $=x$ & $F P_{-} R S S I_{2, N}$ \\
\hline 3 & $\mathrm{x}_{3}, \mathrm{y}_{3}$ & $F P \_R S S I_{31}$ & $F P \_R S S I_{32}$ & $m$ & $F P \_R S S I_{3, N}$ \\
\hline 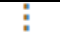 & 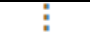 & 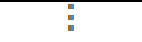 & 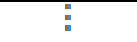 & 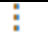 & 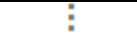 \\
\hline$\overline{\boldsymbol{i}}$ & $x_{i}, y_{i}$ & $F P_{-}{ }_{R S S I_{i 1}}$ & $F P \_R S S I_{i 2}$ & $x$ & $F P_{2} R S S I_{i, N}$ \\
\hline
\end{tabular}

\section{Offline Phase}

Offline phase is when a fingerprint map is created based on surveying from $R S S I A P_{N}$ existing. Each fingerprint has a unique RSSI matrix. The fingerprints will be constructed using the measured RSSI value at certain coordinates. RSSI value can be obtained by war walking using smart phones with a pre-installed mobile application capable of detecting RSSI. $R P$ is marked in the indoor map as seen in Fig. 2. This will serve as the database for identifying a position based solely from RSSI value.

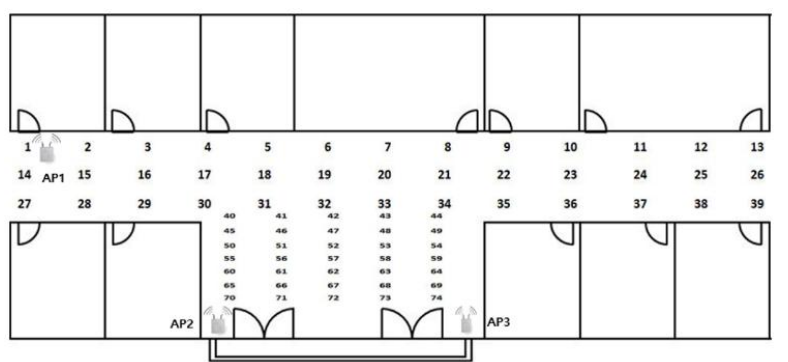

Fig. 2. Generated fingerprint map based during the offline phase to serve as the RSSI database.

\section{Online Phase}

Online Phase is when the active smartphones receive the RSSI from the $A P_{N}$ and these signals will be matched with the fingerprint map database. The user position will be estimated based on the nearest value of RSSI from the RPs as seen in Fig. 3. In this phase, mobile users will freely walk within the indoor environment without knowing the fingerprint map while the received RSSI is matched with the database.

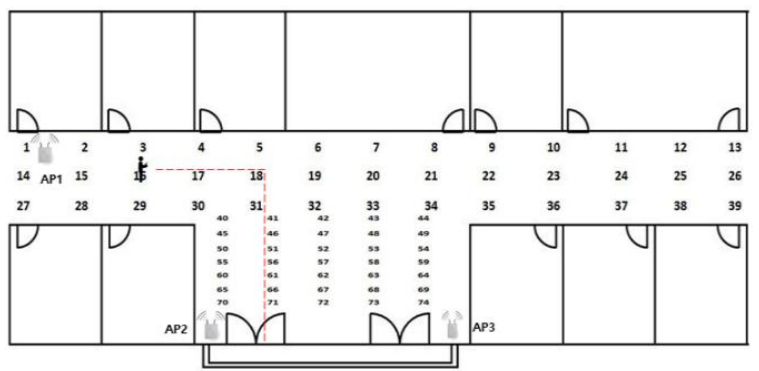

Fig. 3. A scenario of mobile user trajectory and the nearest RPs on the fingerprint map.

\section{EXPERIMENTSAND RESULTS}

The $1^{\text {st }}$ floor on the engineering building of our university was used as a testbed for experiments with a covered area of
$32 \mathrm{~m} \times 32 \mathrm{~m}$. Two points are marked in the map with the start point as green and end point as red as seen in Fig.4. The fingerprint map of the lobby is generated using 74 RPs of about an area of $1 \mathrm{~m}^{2}$. The experiment is done by the mobile user holding a smartphone while walking inside the lobby.

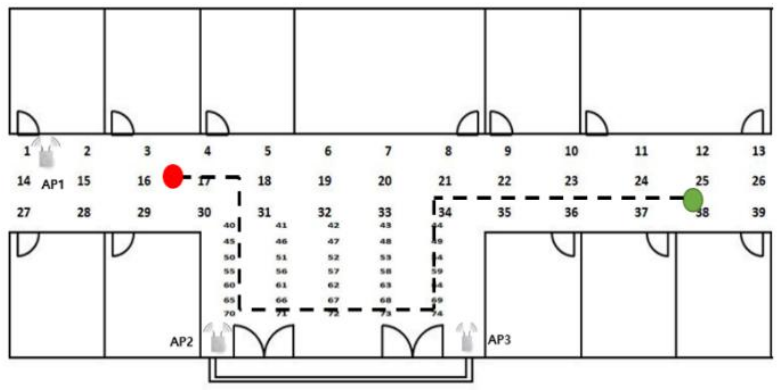

Fig. 4. Layout of the floor plan and the path taken by the mobile user.

After the experimentation, accelerometer sensor data were gathered and processed to estimate the number of steps taken. Low pass filter was applied to reduce noise affecting acceleration. The detected number of steps are marked as red circle while the raw acceleration is marked in red line. The filtered acceleration in blue shows a less fluctuating acceleration compared to the raw data. This helped in detecting the number of steps by disregarding the rise in acceleration due to noise as seen in Fig. 5.

Orientation and heading information are estimated with the use of gyroscope and magnetometer sensors. In the experiment, this data is combined with the number of steps to determine the distance traveled and the direction of the mobile user. An estimated 49 steps were detected which is identical to the 50 steps taken in the experiment. The results are shown in Fig. 6.

The final step in this experiment is to estimate the trajectory and compare it to the actual route taken. Based on the global coordinate frame, the starting point is assigned at zero and oriented to the north. The estimated trajectory showed very identical result with the desired trajectory until the fourth turn where it was slightly longer compared to the original. The overall distance covered shows a high accuracy which is within the experimental testbed as shown in Fig. 7.

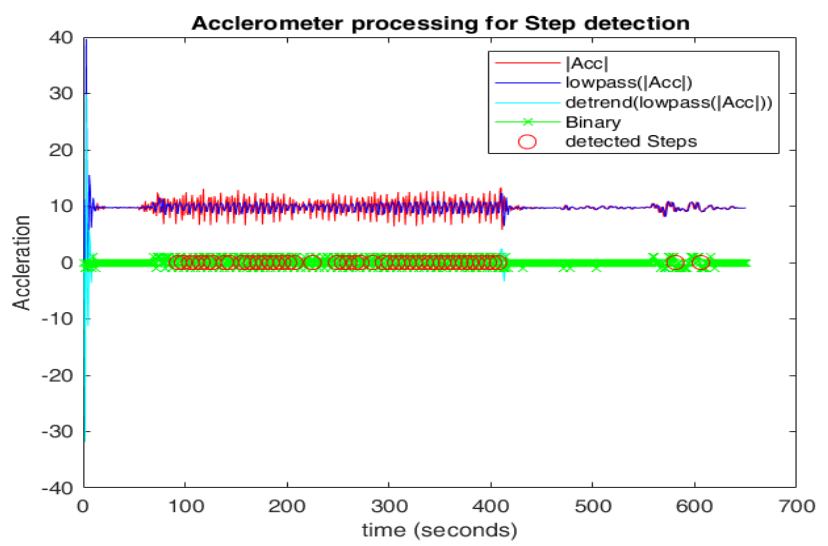

Fig. 5. Estimated number of steps with respect to detected acceleration. 


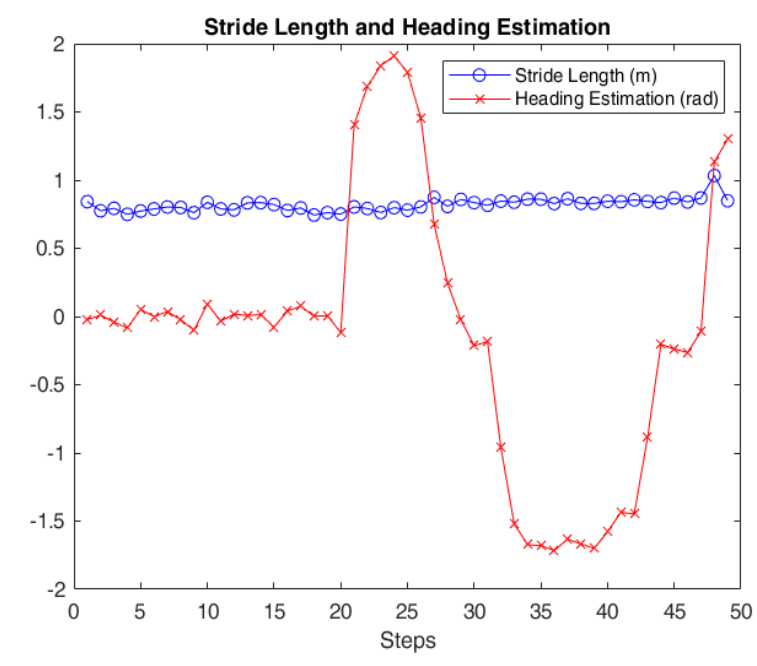

Fig. 6. Stride length based on changes in acceleration and orientation.

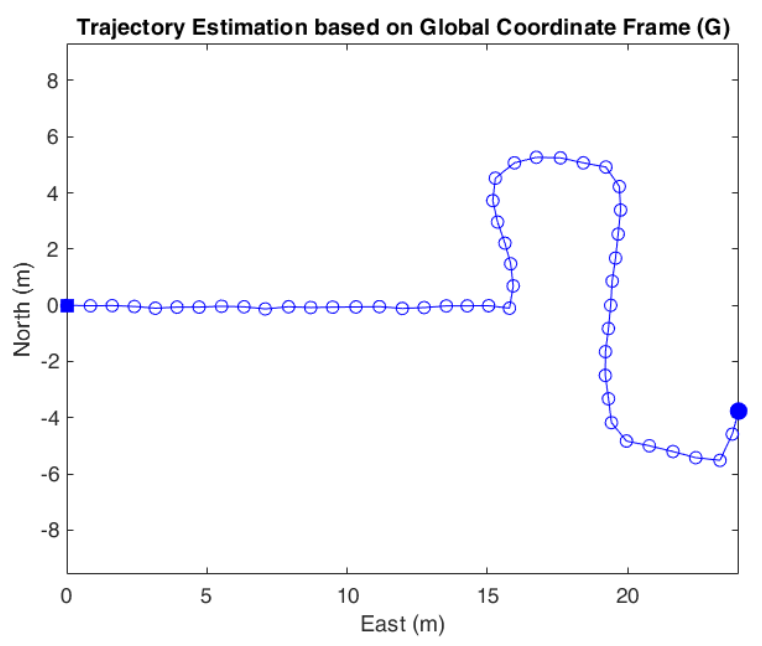

Fig. 7. Estimated taken trajectory from start to end.

\section{CONCLUSION}

This paper proposed the hybrid indoor positioning system that collaborating Wi-Fi RSSI values together with dead reckoning (DR) sensor readings. Two main components of the algorithm use Wi-Fi signals and PDR for smartphone sensor readings. By incorporating the number of steps to take and the total distance to be traveled, this will increase the parameters to work with fusion algorithm which predicts the optimal path to the target. Further study on PDR will be made to determine the orientation of mobile users and to also make an accurate mapping of the movements with the help of the fusion algorithm to improve the positioning accuracy. In addition, the effect of noise interference on sensors and the accumulated errors resulting from walking should be required to generate higher indoor positioning performance.

\section{ACKNOWLEDGMENT}

This work was supported by the BB21+ Project in 2019.

\section{REFERENCES}

1. ZA. Deng, G. Wang, D. Qin, Z. Na, Y. Cui, J. Chen, “Continuous Indoor Positioning Fusing WiFi, Smartphone Sensors and Landmarks," Sensors, 16(9), pp.1427, Sep. 2016.DOI: 10.3390/s16091427.

2. ZA. Deng, Y. Hu, J. Yu, Z. Na, "Extended Kalman filter for real time indoor localization by fusing WiFi and smartphone inertial sensors," Micromachines, 6(4), pp.523-543, 2015. doi:10.3390/mi6040523.

3. Ling-Feng Shi. et al., "A Fusion Algorithm of Indoor Positioning Based on PDR and RSS Fingerprint," IEEE Sensors Journal, vol.18, Issue23, pp.9691-9698, 2018. DOI: 10.1109/JSEN.2018.2873052.

4. Li. Zengke, Liu. Chunyan, Gao. Jingxiang, Li. Xin, "An Improved WiFi/PDR Integrated System Using an Adaptive and Robust Filter for Indoor Localization,” Int. J. Geo-Information, 5(12):224, pp.224-231, 2016. DOI: 10.3390/ijgi5120224.

5. Axel Barrau, "Non-linear state error based extended Kalman filters with applications to navigation," Doctoral dissertation, Mines Paristech, Dec. 2015.

6. P. Bahl and V. Padmanabhan, "RADAR: an in-building RF-based user location and tracking system," in Proc. INFOCOM'00: Nineteenth Annual Joint Conference of the IEEE Computer and Communications Societies, Tel Aviv, Israel, vol.2, pp.775-784, 2000. DOI 10.1109/INFCOM.2000.832252.

7. S. Sen et al., "Precise Indoor Localization Using PHY Layer Information," Proc. 10th ACM Workshop. Hot Topics in Networks, Article no.18, Nov. 2011. DOI: 10.1145/2070562.2070580.

8. M.H. Afzal, V. Renaudin,G. Lachapelle, "Assessment of indoor magnetic field anomalies using multiple magnetometers," In Proceedings of the 23rd International Technical Meeting of The Satellite Division of the Institute of Navigation (ION GNSS 2010), Portland, OR, USA, pp.21-24,Jan. 2010

9. Y. Kim,Y. Chon,H. Cha, "Smartphone-based collaborative and autonomous radio fingerprinting," IEEE Trans. Syst. Man and Cybern. C Appl. Rev.,vol.42, Issue 1, pp.112-122, Dec. 2010. DOI: 10.1109/TSMCC.2010.2093516.

10. H. Wang,S. Sen, A. Elgohary,M. Farid,M. Youssef,R. R. Choudhury, "No need to war-drive: unsupervised indoor localization," In Proceedings of the 10th International Conference on Mobile Systems, Applications, and Services (MobiSys'12), Low Wood Bay, Lake District, UK, pp.197-210, Jun. 2012. DOI: 0.1145/2307636.2307655.

11. LH. Chen, EH. Wu, MH. Jin, GH. Chen, "Intelligent fusion of Wi-Fi and inertial sensor-based positioning systems for indoor pedestrian navigation,'IEEE Sensors Journal, vol.14, Issue 11, pp.4034-4042, Nov. 2014. DOI: 10.1109/JSEN.2014.2330573.

12. Z. Tian, Y. Jin, M. Zhou, Z. Wu, Z. Li,"Wi-Fi/MARG integration for indoor pedestrian localization," Sensors, 16(12), Dec. 2016. DOI $10.3390 / \mathrm{s} 16122100$.

13. Boney A. Labinghisa and Dong Myung Lee, "Hybrid Indoor Positioning System using Wi-Fi and PDR in Smartphone," Proceedings of 2019 International Research Conference on Innovation, Technology and Sustainability (IRCITS). vol.6, no.1, pp.508-509, Jan. 2019.

14. SJ. Julier and JK. Uhlmann, "New extension of the Kalman filter to nonlinear systems," In Signal processing, sensor fusion, and target recognition, International Society for Optics and Photonics. pp.182-194, Jul. 1997. https://doi.org/10.1117/12.280797.

15. Eric A. Wan and Rudolph Van Der Merwe, "The unscented Kalman filter for nonlinear estimation," In Proceedings of the IEEE 2000 Adaptive Systems for Signal Processing, Communications, and Control Symposium, pp.153-158, Oct. 2000. DOI 10.1109/ASSPCC.2000.882463.

16. Tim Bailey, Juan Nieto, Jose Guivant, Michael Stevens, and Eduardo Nebot,"Consistency of the ekf-slam algorithm," 2006 EEE/RS. International Conference on Intelligent Robots and Systems, pp.3562-3568, Oct. 2006. DOI: 10.1109/IROS.2006.281644.

17. Olivier Cappé, Simon J Godsill, and Eric Moulines, "An overview of existing methods and recent advances in sequential monte carlo," Proceedings of the IEEE, vol.95, Issue 5, pp.899-924, Jul. 2007. DOI: 10.1109/JPROC.2007.893250.

18. A. Sakai, D. Ingram, J. Dinius, K. Chawla, A. Raffin, A. Paques, "PythonRobotics: a Python code collection of robotics algorithms,"Sep. 2018 


\section{AUTHORS PROFILE}

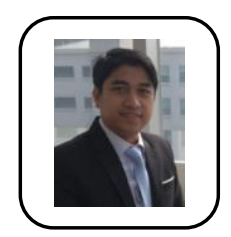

Name: Boney A. Labinghisa

Born: February 26, 1989

E-mail:blabinghisa@yahoo.com

Mobile: +82-10-5942-1566

Boney A. Labinghisa received his B.S. degree in Electronics Engineering from Central Philippine University, Philippines in 2011. He worked in the telecommunications industry for over 2 years and continued his graduate studies on 2016. He completed his M.S degree in Dept. of Computer Media Engineering in Tongmyong University, Busan, Republic of Korea on 2018. $\mathrm{He}$ is now pursuing his Ph.D. in Tongmyong University while expanding his studies. His research interests are in the area of wireless communications, sensor networks, localization system, machine learning, deep learning and mostly on indoor navigation.

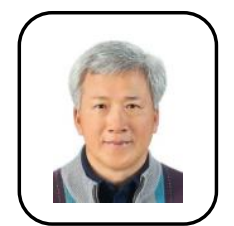

Name: Professor Dong Myung Lee

Born: June 07, 1958

E-mail: dmlee@tu.ac.kr

Mobile: +82-10-9410-9681

Prof. Lee had worked as a principal member of research staff at Electronics and Telecommunications Institute (ETRI) that located in Taejon during 18 years. In ETRI, he had participated to some national projects for developing some systems that running various network protocols based on the OSI model during 1982-1988. After then, he had participated to the CDMA cellular system project, and had designed and implemented the call processing and handoff functions that are a key role at the Base Station in the CDMA cellular system during 1991-1998. He had also worked the fundamental researches for the 3rd generation wireless system and joined a project to develop the IMT-2000 system during 1996-2000. In the year 2000, he had transferred to the department of computer engineering in Tongmyong University. He has been teaching network related courses (computer network, network programming) in undergraduate school and some advanced courses in graduate school (mobile computing and high speed communication network) in Tongmyong University since 2000 . He has been now working about the researches on sensor based localization algorithms with graduate school students by several projects 